\title{
Recycle of Zincates and Aluminum to Fed Zn-Air Fuel Cells ${ }^{\dagger}$
}

\author{
Patrizia Bocchetta $^{1, *}$, Donato Cannoletta ${ }^{1}$, Filippo Selleri ${ }^{2}$ \\ 1 Dipartimento di Ingegneria dell'Innovazione, Università del Salento, via Monteroni, 73100 Lecce, Italy; \\ patrizia.bocchetta@unisalento.it (P.B.); donato.cannoletta@unisalento.it (D.C.); \\ 2 Eco Salento S.n.c., Via Preti di Campi, 17, 73010 Lequile (LE); info@ecoanalisisalento.it (F.S.); \\ * Correspondence: e patrizia.bocchetta@unisalento.it; \\ $\dagger$ Presented at Materials Chemistry and Physics (Materials Chemistry 2020) - International e-Conference
}

Received: 16.09.2020; Revised: 20.09.2020; Accepted: 24.09.2020; Published: 27.09.2020

\begin{abstract}
It is well known that the Zn-air battery dept of discharge is mainly governed by the electrochemistry of zinc anode in concentrated alkaline solutions leading to undesired precipitation of insulating zinc oxide at the electrode surface due to progressive increase in zincate concentration. Among the different approaches adopted to overcome this problem, mechanically and electrically rechargeable as well as refuelable systems have been deeply studied for automotive and stationary applications. In a refuelable $\mathrm{Zn}$-air battery, the alkaline aqueous electrolyte is pumped into the cell, either carrying $\mathrm{Zn}$ electroactive fuel or flowing through a packed bed of $\mathrm{Zn}$ particles. In a previous paper, we adopted a mechanically refuelable tapered-end flow $\mathrm{Zn}$-air fuel cell with $\mathrm{Zn}$ micro-spheres, and we studied the effect of electrolyte aging on the behavior of the $\mathrm{Zn}$ anode during battery discharge. The results have shown that the cell potential decrease in the battery discharge curve is mainly due to the anodic overpotential increase, as evidenced by means of EIS (Electrochemical Impedance Spectroscopy) measurements. Given that this effect can be attributed to the physicochemical modifications induced by the $\mathrm{Zn}$ passivation occurring at higher zincates concentration, the performance of the battery system can be notably improved by a relevant regeneration of spent alkaline zincate bath.

Here, we propose a novel method to recover zinc from alkaline zincate baths by using aluminum electrodes. Aluminum metal and zincate ions give place to a single-displacement reaction producing a layer of zinc on the $\mathrm{Al}$ surface. The deposits of zinc metal on aluminum foils have been studied by means of X-ray diffraction (XRD), scanning electron microscopy (SEM), and the electrochemical process has been followed by open circuit measurements in order to put the basis of future developments.
\end{abstract}

Keywords: zinc air fuel cell; displacement reaction; zincate regeneration.

(C) 2020 by the authors. This article is an open-access article distributed under the terms and conditions of the Creative Commons Attribution (CC BY) license (https://creativecommons.org/licenses/by/4.0/).

\section{Funding}

This research received no external funding.

\section{Acknowledgments}

This research has no acknowledgment. 


\section{Conflicts of Interest}

The authors declare no conflict of interest. The funders had no role in the design of the study; in the collection, analyses, or interpretation of data; in the writing of the manuscript, or in the decision to publish the results. 